\title{
协同演化概念的发展、使用误区与研究证据
}

\author{
童泽宇，黄双全* \\ 华中师范大学生命科学学院进化与生态学研究所, 武汉 430079 \\ *联系人, E-mail: hsq@mail.ccnu.edu.cn
}

收稿日期：2018-10-16; 接受日期：2018-12-13; 网络版发表日期：2019-02-18

国家自然科学基金(批准号: U1402267, 31730012, 31800192)和博士后创新人才支持计划(批准号: BX201600059)资助

\begin{abstract}
摘要 自然选择是生物演化的重要动力, 而协同演化的理念使人们认识到, 互作物种之间彼此交互的选择也可以 驱动物种发生演化. 自1964年正式提出以来，协同演化的概念经历了充分的发展与拓展，但使用误区也多种多样; 虽然不断有研究验证一些生态互作关系的确是协同演化, 但质疑协同演化关系真实性的论断也时有报道. 为了理 清协同演化概念中存在的认识误区，我们尝试提出避免误区的3条简要准则，即具有互作关系、交互选择、协同 系统发生. 通过文献计量学分析量化了协同演化概念的发展，显示协同演化概念向非进化生物学领域的迅猛拓 展，同时统计结果也揭示了人们对多个易与协同演化概念混淆的术语的使用情况尚在合理的范围。依据生态关 系，我们总结了现有的发生协同演化的互作系统及证据; 介绍了协同演化的地理镶嵌理论的内容与案例. 对协同 演化概念的历史梳理、文献计量、证据收集和前沿发展方向的介绍, 有助于促进人们对协同演化的深入理解, 避 免误用术语, 期望为后续协同演化方向的研究提供有益的参考.
\end{abstract}

关键词 生态关系, 相互作用, 协同演化, 协同演化的地理镶嵌, 演化

演化与协同演化: 经典的演化(evolution), 是居群 (种群、族群)基因型对环境的适应, 体现自然选择(natural selection)的结果，也即亲子代居群基因频率的变 化; 协同演化(coevolution)是演化的子集, 主要驱动力 来自于相互作用的物种之间, 体现交互选择(reciprocal selection)的结果.

相互作用与协同演化：存在于地球上的不同物 种、不同个体之间因为对空间资源的占据，不可避免 会发生相互作用(interaction)。“相互作用”更多体现为 生态学描述，因为相互作用可发生在时间维度上的任 意一个点，自身并不包含连续的时间属性. “协同演
化”这一术语用于描述两个或更多物种交互地影响彼 此的演化. 与“相互作用”相比, “协同演化”至少包含了 3方面信息: 相互作用的状态、时间维度、相互作用的 结果.

\section{1 协同演化概念的发展历程}

生物体在环境中不是孤立存在的, 而是在与其他 生物体的相互作用中不断变化、发展. 协同演化思想 的倠形源起于对生物体之间相互作用最基础形式的思 考：一个主体与另一个主体之间的相互影响、共同

引用格式: 童泽宇, 黄双全. 协同演化概念的发展、使用误区与研究证据. 中国科学: 生命科学, 2019, 49: 421-435

Tong Z Y, Huang S Q. The development, misuse and evidence of the concept "coevolution" (in Chinese). Sci Sin Vitae, 2019, 49: 421-435, doi: 10.1360/ N052018-00221 
变化.

早在1859年，达尔文在《物种起源》中以协同适 应(co-adaptation)的角度描述了植物与传粉者之间的 相互作用 ${ }^{[1]}$. 真正意义上的协同演化概念由Ehrlich和

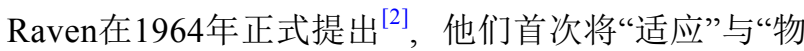
种形成”链接到互作物种中. 在他们的研究系统中, 粉 蝶幼虫以宿主植物为食, 而宿主植物产生的有毒次级 代谢产物影响粉蝶幼虫的取食行为, 粉蝶幼虫对毒性 的抗性则反过来影响植物毒性的演化. 依据这一特性, Ehrlich和Raven还提出了逃脱-辐射协同演化(escaperadiate coevolution)模型，即被子植物普遍遭受着严重 的啃食, 一旦植物突变产生有毒次级代谢产物影响植 食昆虫的取食, 则可发生适应辐射, 同时, 植食昆虫如 果突变出抗毒性, 则有此突变的昆虫将比其他植食昆 虫更具优势, 从而也可发生适应辐射 ${ }^{[2]}$.

初期狭义的协同演化定义，将协同演化互作限定 在物种与物种之间, 通常被称作成对协同演化 (pairwise coevolution) ${ }^{[3]}$ 或专性协同演化(obligate coevolution $)^{[4]}$. 在自然界中, 物种间互作极少构成一对一的种 间关系，事实上一对多或多对多的互作形式更为常见， 如一种植物可能需要同时抵御多种啃食者的啃食, 或 需要多种动物为其传粉及扩散种子, 而一种动物也常 可以为多种植物提供服务，以及捕食多种不同的猎物， 因而, 弥散协同演化(diffuse coevolution)或团体协同演 化 (guild coevolution)得以提出 ${ }^{[3,5]}$. 弥散协同演化可用 来解释某一个或多个物种的特征受到多个其他物种特 征的影响而产生的相互演化现象, 包括植物受多种害 虫的取食而产生的物理和化学防御机制, 以及昆虫演 化出能降解多种植物有毒物质的能力等.

\section{2 协同演化概念使用中的误区}

协同演化的现代定义是：互作有机体间因相互适 应而共同发生演化的过程 ${ }^{[6]}$. 协同演化能在许多生物 阶层上发生. 微观上，协同演化可以是氨基酸间的关 联性突变 ${ }^{[7]}$; 宏观上, 协同演化体现为环境中不同物种 的共变特征 ${ }^{[8]}$. 协同演化的主体之间都交互选择彼此, 从而影响各自的演化.

对于协同演化概念使用的误区主要有3种: 一是将 单向作用误解为双向互作, 即将生态互作关系中, 只有 一方发生改变, 而另一方几乎不变的情况误认为是发
生了双向改变. 二是将生态互作关系直接等同于协同 演化关系, 这些生态互作关系包括协作(cooperation)、 互惠(mutualism)、互利共生(symbiosis)、协同适应 (coadaptation)等. 例如, 蜜蜂与很多开花植物是互惠的 生态关系，但并不表明蜜蜂与这些开花植物的每一种 都发生了协同演化. 三是将“演化”混同于“变化”, 完全 忽略“协同演化”中“演化”的生物学意义，这一误区产 生的根源是“coevolution”这一单词原本就有“随着时 间发生变化”的含义. 然而, 生物学意义上的“演化”并 不仅仅是任意主体随时间发生的变化, 其变化主体为 生物居群的可遗传性状, 且时间变化以世代延续为 基础.

\section{1 生物与非生物之间、非生物与非生物之间是 协同演化吗?}

(1) 人与自然. 一方面, 在漫长的演化过程中, 自然 选择作用于人类, 人类以及其他各式各样的生物在漫 长的演化过程中, 逐渐对地球环境产生了适应性. 另 一方面，人类是否在选择自然环境？虽然自工业革命 以来，人类的生产力和科技水平呈现爆炸式的增长, 对地球打下了深刻的烙印 ${ }^{[9,10]}$, 带来了一系列如全球 气候变暖、臭氧层空洞、水体污染和荒漠化等问题. 然而, 地球环境本身并不需要去适应人类对环境产生 的改变.

(2) 生物圈与早期地质环境. 在地球形成早期, 地 表温度高, 大气含氧量低; 早期的生物圈侵蚀高山、固 定水土、防止风化、阻隔沙尘，大幅改变了地球表面 的面貌, 使其变得更加适宜生物居住. 许多观点认为, 早期地质环境作用于生物圈, 使其更能适应恶劣环境 的影响; 早期的生物圈也作用于地质环境, 使其更加 适宜生物居住 ${ }^{[11,12]}$. 可以清楚地看到, 两者之间没有 必然的选择压力作用，它们仅仅只是相互作用，而不 是相互选择，同样也不是演化. 固然生物圈发生了演 化, 而地质环境却并没有必要随之发生改变. 它们之 间并不是生物学协同演化的关系，而仅仅是发生了相 互作用.

(3) 共存星系的演变. 星系的确存在“演化”的过程, 且不同的星系之间、星系与黑洞之间的引力作用, 在 星系形成的过程中会影响到彼此的演化过程 ${ }^{[13,14]}$. 显 然，宇宙学中“协同演化”这一术语的使用，其含义并 不是对应进化生物学意义上的“协同演化”. 


\section{2 支持协同演化的证据}

目前, 支持协同演化所需的证据至少包含 3 类 ${ }^{[3,6,15]}$ (图1):

（i ）实地观察确定生态互作关系. 尽管演化是一 个漫长的过程，现在人们已经可以根据广泛、长期的 观察来确定两个互作类群之间是否存在着协同互作的 关系.

(ii) 实验证据确定彼此有选择作用. 选择是演化 的基本要素, 通过选择, 居群中突变的等位基因频率才 得以固定下来. 同样, 在协同互作的系统中, 需要通过 实验证据确定互作的类群之间存在双向的选择作用.

(iii) 系统发生证据确定协同系统发生. 系统演化 树是体现生物类群宏观演化样式的直观手段. 如果发 生互作的类群(或物种), 其系统发生树拓扑结构表现 一致, 即可作为两类群间发生协同演化的有效证据.

\section{3 文献计量分析}

\section{1 协同演化概念发展中的问题}

在协同演化概念不断发展、经受误用的过程中, 有两个显而易见、颇具意义的问题尚未定量分析： （i ）协同演化概念是如何向非进化生物学领域拓展 的; (ii) 协同演化概念的近义概念，其应用情况是怎 样的.

\section{2 文献计量分析方法}

为了解决这两个问题，本文采用文献计量学手段， 以科瑞唯安信息服务有限公司(Clarivate Analytics，原 汤森路透知识产权与科技事业部)的Web of Science信 息平台所提供的Web of Science核心合集作为数据源.

对于问题( i ), 我们先使用检索式 $\mathrm{TS}=$ (“coevolution*” OR “co-evolution") OR TI=(“coevolution” OR “co-evolution”)检索1964 2017年间所有与协同演化有 关的文献，以文献类型“文章(Article)”进行精炼，然后 以学科类别“进化生物学(Evolutionary Biology)”进行 精炼, 获得文献2821篇, 以学科类别排除“进化生物学 (Evolutionary Biology)”进行精炼, 获得文献11371篇.

对于问题( ii), 首先, 我们依据前人经验 ${ }^{[3,6]}$, 总结 了6个易被混用的、协同演化的近义概念：相互作用 (interaction)、相互影响(interplay)、互惠(mutualism)、互利共生(symbiosis)、协作(cooperation)、协同

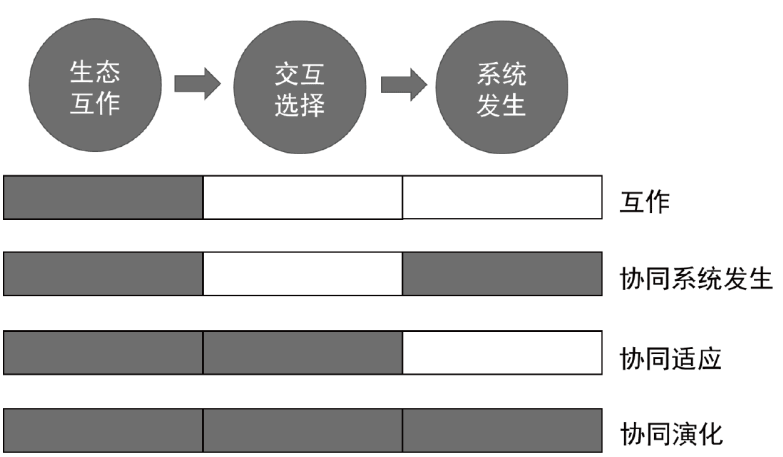

图 1 互作类群间协同演化关系的条件. 3个递进条件下方 的灰色条块代表满足条件、具有证据, 白色条块代表不满足 条件、证据缺失. 不同条块的组合代表的进化生态关系列于 右侧

Figure 1 Conditions for estimating the coevolutionary relationship between interacting groups. Gray bars represent conditions satisfied and evidence present, while white bars represent conditions unsatisfied and evidence absent. Different combinations of bars represent different ecological evolutionary relationships, and are listed to the right

适应(coadaptation). 其中, “相互作用”和“相互影响”的 意义相同，都是指两个或更多主体对彼此造成影响的 行动; “互惠”是两个主体间, 彼此的适合度因对方的 行为而获益的关系; “互利共生”是更为紧密的互惠关 系，是两个主体间紧密的、长期的互作; “协作”是主 体间为了共同利益而一起行动的关系; “协同适应”是 两个或更多主体以一对或一群的形式，发生适应性改 变的过程. 简而言之, “互惠”、“互利共生”和“协作”都 是生态上“相互作用”、“相互影响”的形式，而生物类 群间只有经历“协同适应”的过程，才可能实现“协同演 化”. 然后, 我们为每个术语构建检索式: ( i ) TS= (“coadapt*” OR “coadapt*”) OR TI=(“co-adapt* OR co-adapt*"); ( ii ) TS=(“cooperat*” OR “co-operat*”) OR $\mathrm{TI}=$ (“cooperat*" OR "co-operat*"); (iii) $\mathrm{TS}=$ ("interact*”) OR TI=(“interact*”); (iv) TS=(“interplay*”) OR $\mathrm{TI}=$ (“interplay*”); ( v ) TS=("mutualis*”) OR TI=(“mutualis*”); (vi) TS=(“symbio*”) OR TI=(“symbio*”). 检 索1964 2017年间的所有文献，以文献类型“文章(Article)”和学科类别“进化生物学(Evolutionary Biology)” 进行精炼, 得出coadaptation为 231 篇, cooperation为 1585 篇, interaction为 10182 篇, interplay为 555 篇, mutualism为 1143 篇, symbiosis为 1734 篇.

\section{3 文献计量分析结果与意义}

（1）协同演化概念是如何向非进化生物学领域的 
拓展. 虽然协同演化的概念自1964年就已正式提出, 但 直到1991年，协同演化概念在文章中的应用才体现出 明显增长的趋势：在进化生物学领域，每年文章数从 1990年的6篇跃升为1991年的22篇; 而在非进化生物 学领域，每年文章数从1990年的16篇跃升为1991年的 78篇. 从1990年到2017年, 在进化生物学领域, 全球每 年与协同演化有关的文章发表数量从6篇增长至 191 篇, 平均增长率为6.3篇/年, 而在非进化生物学领域, 全 球每年与协同演化有关的文章发表数量从 16 篇爆发增 长至1007篇，平均增长率高达34.4篇/年．从1964年到 2017年，各年发表文章的数量与年份的关系符合指数 分布，曲线拟合的结果也表明，与“coevolution”概念有 关的文章年发表数量，在非进化生物学领域的增长率 远高于进化生物学领域(表1)。这一结果，一方面说明 协同演化概念在常规领域的运用正在不断增长，另一 方面也表明，协同演化概念已经广泛拓展至非进化生 物学领域，且在其他领域的应用、发展速度还要超过 原本所处领域中的表现．甚至可以说，协同演化概念 应用的快速增长，更多体现在非进化生物学领域. 由 于非进化生物学领域的协同演化概念，其中“演化”已 经脱离“演化”的生物学意义，这样的拓展也为协同演 化概念的误用埋下了伏笔.

（2）协同演化近义概念的使用情况. 在所有术语年 发文量增长曲线拟合中, 与幂函数相比, 指数函数的拟 合度更高、标准误更低(表1)，因而在本文中，指数函 数更适合用于评估术语的发展情况. 在所有本文探讨 的协同演化概念的近义词术语中，“interaction”这一术 语的发展速度远超其他术语，甚至超过“coevolution”. “interaction”的起始增长时间为1991年：1990年该术语 发文量为23篇，而1991年则一跃变为107篇; 从1990年 到2017年，在进化生物学领域，与“interaction”有关文 章的每年发文数从 23 篇增长为 868 篇，平均增长率高 达28.2篇/年，其指数函数拟合曲线增长率也远高于其 他本文探讨的近似术语(表1). 与之相比, “coevolution” 的增长速度则稍显平缓，从1990年的6篇稳步增长至 191篇，平均增长率为6.3篇/年. “cooperation”, “mutualism”和“symbiosis”表现平缓, 开始出现明显增长的年份 均为1991年, 平均年增长率分别为: 3.2, 3.5 和5.7篇/年. 此外, “interplay”和“coadaptation”的增长率较低, 分别为 1.8 和 0.1 篇/年. 进化生物学领域“interaction”概念的使 用远超其他概念, 表明在进化生物学领域, 人们越来越 关注不同物种间的“互作(interaction)”, 而不再是将目光 局限于单一物种适应于环境而发生的进化转变, 从而 以更为“生态”的角度来探讨“进化”。“Interaction”的热 门程度远高于 “coevolution”, 也说明了并非所有对于互 作物种的演化研究，都上升到了对“协同演化”进行探 讨的高度. “Coevolution”概念的使用高于其他下级附 属概念，说明人们对诸如“coadaptation”, “cooperation”, “interplay”, “mutualism”和“symbiosis”等概念的探讨, 仍 处于协同演化概念的范畴内, 概念运用尚属合理.

\section{4 协同演化的系统与证据}

协同演化要求相互作用的双方在生态上和演化上

表 1 “协同演化”的近似术语, 其相关主题每年发文量 $(y)$ 随时间 $(x)$ 增长的曲线拟合参数 ${ }^{a)}$

Table 1 Parameters of curve fitting of publications per year $(y)$ against time $(x)$ of similar terminologies of "coevolution",a)

\begin{tabular}{|c|c|c|c|c|c|c|c|}
\hline \multirow{2}{*}{ 术语 } & \multicolumn{3}{|c|}{ 幂函数拟合 } & \multicolumn{3}{|c|}{ 指数函数拟合 } & \multirow{2}{*}{ 增长系数 ${ }^{\mathrm{b}}$} \\
\hline & 公式 & 拟合度 $\left(R^{2}\right)$ & 标准误 & 公式 & 拟合度 $\left(R^{2}\right)$ & 标准误 & \\
\hline Coevolution (进化生物学) & $y=0.04 x^{1.89}$ & 0.679 & 1.18 & $y=0.38 \mathrm{e}^{0.13 x}$ & 0.937 & 0.52 & 0.13 \\
\hline Coevolution (非进化生物学) & $y=0.02 x^{2.50}$ & 0.773 & 1.22 & $y=0.42 \mathrm{e}^{0.16 x}$ & 0.956 & 0.54 & 0.16 \\
\hline Coadaptation & $y=0.19 x^{0.92}$ & 0.626 & 0.62 & $y=0.59 \mathrm{e}^{0.06 x}$ & 0.861 & 0.39 & 0.06 \\
\hline Cooperation & $y=0.05 x^{1.69}$ & 0.633 & 1.16 & $y=0.33 \mathrm{e}^{0.11 x}$ & 0.911 & 0.57 & 0.12 \\
\hline Interaction & $y=0.02 x^{2.43}$ & 0.778 & 1.17 & $y=0.50 \mathrm{e}^{0.15 x}$ & 0.945 & 0.58 & 0.15 \\
\hline Interplay & $y=0.09 x^{1.21}$ & 0.505 & 1.08 & $y=0.32 \mathrm{e}^{0.09 x}$ & 0.845 & 0.61 & 0.09 \\
\hline Mutualism & $y=0.06 x^{1.53}$ & 0.632 & 1.06 & $y=0.37 \mathrm{e}^{0.10 x}$ & 0.912 & 0.52 & 0.10 \\
\hline Symbiosis & $y=0.05 x^{1.69}$ & 0.626 & 1.18 & $y=0.35 \mathrm{e}^{0.12 x}$ & 0.910 & 0.57 & 0.12 \\
\hline
\end{tabular}

a) 所有拟合的 $P$ 值均小于 $0.001 ; \mathrm{b})$ 增长系数是指数函数 $\left(y=a \mathrm{e}^{b x}\right)$ 进行线性转化 $(\ln y=b x+\ln a)$ 后, 曲线的斜率 $b$. 
都紧密相联. 根据获利与受损的情况, 协同演化的研究 系统, 在二元主体互作的情况下, 包含捕食(一方压倒 另一方, 单方获益)、互惠(双方获益)、对抗(势均力 敌, 双方受损)、寄生(一方直接依赖另一方, 单方获 益)、拟态(一方间接依赖另一方, 单方获益). 在自然 状态下, 互作双方的关系并不单一, 在一些广泛研究 的系统中, 互作双方也可同时存在互惠与寄生关系.

\section{1 捕食}

（1）捕食者-被捕食者. 捕食者杀害猎物并同化其 一部分生物量. 为了捕获猎物, 捕食者演化出了更快的 速度、更强大的力量、更强的耐力、更强的咬合力、 偷袭行为、敏锐的感觉、超强的消化能力、对猎物的 评估能力等捕食能力, 而被捕食者演化出了逃跑能 力、防御能力、繁殖能力(超补偿)等一系列反捕食策 略 ${ }^{[16]}$.

捕食-被捕食者互作历史久远. 例如, 研究人员通 过研究一具保存完好的甲龙(Borealopelta markmitchelli)化石, 发现这种甲龙不仅披有带角的骨质装甲和 厚鳞片, 还具有反荫蔽保护色(身体背面颜色较深, 而 腹面颜色较浅)的体色样式, 表明早在中生代, 被捕食 者就受到了捕食者的强烈选择 ${ }^{[17]}$.

捕食者的攻击性和被捕食者的防御性是经典的协 同演化关系, 如非洲坦噶尼喀湖中的腹足纲(Gastropoda)软体动物比其他淡水中的软体动物有更厚的甲壳, 与此同时, 以它们为食的螃蟹比其他地区的淡水蟹有 更粗壮有力的蟹钳 ${ }^{[18,19]}$. 捕食者的毒性和被捕食者的 抗毒性也可能是协同演化的后果, 北美洲的乌梢蛇 (garter snake, Thamnophis sirtalis) 以一种蝾螈(toxic newt, Taricha) 为食, 这种蝾螈可在体表产生特有的神 经毒素(tetrodotoxin, TTX)以抵抗捕食者, 不同地区的 乌梢蛇则相应地演化出对此毒素不同程度的抗性, 在 捕食者与被捕食者间发生了性状的“军备竞赛”, ${ }^{20]}$. 在 美国加利福利亚地区, 有毒性的响尾蛇以地松鼠 (Sciuridae: Xerinae)为食, 地松鼠的血清中演化出相应 的抗毒素以对抗捕食, 不同地区的地松鼠, 其血清中抗 毒素的抗性各异, 而各地区的响尾蛇毒素也依抗毒素 的抗性不同而改变 ${ }^{[21]}$.

(2) 植物-植食者. 与捕食者与被捕食者的关系类 似, 植食者消耗、同化植物的一部分或全部组织, 而植 物演化出防御机制以抵抗或降低植食者的不利影响.
植食者和受到啃食的植物多样化程度很高, 且植食者 在被子植物的多样化中扮演了重要角色 ${ }^{[22,23]}$.

植物与植食者的互作关系由来已久. 例如, 在哥伦 比亚新热带地区森林中, 研究人员在距今约 5800 万年 前的化石叶片上发现有昆虫严重啃食的痕迹 ${ }^{[24]}$. 在巴 西东北部阿拉里普盆地(Araripe Basin), 研究人员通过 分析化石标本, 发现在白严纪早期的有花植物上有昆 虫造成的损伤痕迹 ${ }^{[25]}$.

在与植食者的互作中，植物演化出耐受(tolerance) 或抵抗(resistance)机制以降低植食者的危害. 通过耐受 植食者的机制, 植物即使经受植食者的伤害也不降低 或较少降低适合度, 这既可通过转移植食者的注意, 使 它们啃食较不重要的部位以保护更重要的繁殖器 官 ${ }^{[26]}$, 或是通过自身的快速再生以实现超补偿(overcompensation $)^{[27]}$. 通过抵抗植食者的机制, 植物可降低 植食者的损害程度. 大体上, 抵抗植食者的机制包含颜 色、物理防御、化学防御等方面. ( i ) 颜色方面: 许多 植物幼嫩的、缺乏机械防御的叶片常为红色, 以警示 可能的植食者 ${ }^{[28]}$; 一些植物的叶片拥有隐蔽色(灰色), 使植食者难以辨别并攻击 ${ }^{[29]}$. (ii) 物理防御方面: 许多 植物茎秆和叶片表面有蜡质层、棘刺、表皮毛等结构, 一些组织甚至木质化, 以阻止或降低植食者的摄食 ${ }^{[30]}$. (iii) 化学防御方面: 许多植物可生产并储存生长发育 非必需的次级代谢产物, 包含萜类、酚类、生物碱等, 以各种方式驱赶植食者、毒杀植食者 ${ }^{[31]}$, 或是吸引植 食者的天敌 ${ }^{[32,33]}$ 、阻止植食者对植物的损害 ${ }^{[34,35]}$.

植食者同样对植物的防御措施作出响应. 在植物 与植食者的互作中, 特化的植食者更少受到植物化学 防御物的影响 ${ }^{[27]}$, 一些特化植食者发展出了化解植物 化学防御物的机制 ${ }^{[36,37]}$. 例如, 许多十字花科植物有 硫代葡萄糖苷/黑芥子酶(glucosinolate/myrosinase)防 御机制, 黑芥子酶催化硫代葡萄糖苷生成有毒的异硫 氧酸酯, 但特化植食者鳞翅目昆虫Pieris rapae内脏中 有一种蛋白可以指导水解反应向着生成腈类化合物而 不是异硫氧酸酯(isothiocyanate)的方向进行 ${ }^{[38]}$. 凤蝶科 的Palilio polyxenes适应于在有毒的宿主植物上受食, 因其细胞色素P450单氧酶(monooxygenase)可参与有 毒的呋喃香豆素(furanocoumarins)的脱毒过程 ${ }^{[39]}$.

\section{2 互惠}

(1) 植物与传粉者. 传粉者为植物提供传粉服务, 
使花粉从花药中传递到柱头上, 完成有性生殖, 而植物 为传粉者提供花蜜、花粉等作为报酬.

在宏观尺度上, 有花植物与传粉者之间被认为是 协同演化的 ${ }^{[0]}$. 例如, 有花植物自白严纪中期(距今约 1 亿年)开始辐射演化，并随后占据植物界主导地位 ${ }^{[41,42]}$, 而现存蜂类主要分支同样起源于白严纪中-晚期 ${ }^{[40]}$.

从系统发生的角度来看, 被子植物基部类群即有 互作的传粉昆虫. 五味子科(Schisandraceae)是被子植 物基部类群ANITA中最大的科，一项 9 年跨度的研究 探讨了 21 种五味子科植物与瘦蚊科(Cecidomyiidae, Diptera)昆虫的协同演化关系，其中夜间传粉的瘦蚊将 卵产在花中, 幼虫以花的分泌液为食, 而分子钟分析揭 示出, 五味子科植物与产卵瘘蚊在中新世早期就已开 始互作 ${ }^{[43]}$.

花部特征的各个方面都体现出对特定传粉者或传 粉功能群的适应, 与此同时, 特定的传粉者也演化出相 应特征以适应花部特征发生的改变.

(i ) 花外形结构. 花细长的花冠管或蜜距, 被认为 是对长吻传粉者的适应. 例如, 在尼泊尔, 一种紫色象 牙参(Roscoea purpurea)在 5 个不同地区的居群有不同 的花冠管长, 而各地对应有不同吻长的长吻蝇类传粉 者 ${ }^{[44]}$. 在南非有一种长吻蝇类传粉者Prosoeca ganglbaueri (Nemestrinidae), 其吻长可达20 50 mm, 以一 种玄参科植物Zaluzianskya microsiphon蜜距中的蜜为 食, 在不同居群, Z. microsiphon的蜜距长度各异, 而蜜 距越长的居群, 传粉蝇类的吻也越长 ${ }^{[45,46]}$. 马达加斯加 有大约 30 种长距的兰花物种和 7 种长吻天蛾, 达尔文曾 预测过该地彗星兰(Angraecum sesquipedale) 长达 $33 \mathrm{~cm}$ 的蜜距是与具有相应长度吻长天蛾的协同适应, 随后人们在彗星兰上的确发现了超过 $30 \mathrm{~cm}$ 吻长的蛾 类 ${ }^{[47]}$. 最近，研究人员使用化石标定的分子钟测定得 出马达加斯加长吻天蛾和长距兰花从非洲大陆分化出 来的时间高度匹配, 均为 750 万年左右 ${ }^{[48]}$.

(ii) 花色. 不同植物花的颜色(反射光谱)与各类特 化传粉者的颜色视觉之间存在关联 ${ }^{[49,50]}$. 有花植物演 化出多种多样的颜色以吸引不同的传粉者，而传粉者 的视觉特性也同样选择作用于植物 ${ }^{[51 ~ 53]}$. 例如, 蜂类 是三基色视觉系统，对紫外线、蓝色和绿色波段的光 很敏感 ${ }^{[54]}$, 而鸟类是四基色视觉系统, 对红光更敏 感 ${ }^{[5,56]}$. 此外，许多被子植物的花瓣上具有纳米级别 的不规则结构，可导致光的干涉，生成短波长的散射
光，形成结构性颜色，促进对昆虫传粉者尤其是熊蜂 的吸引 ${ }^{[57]}$.

(iii) 花气味. 植物的花可以合成并散发气体化合 物以吸引传粉者 ${ }^{[58,59]}$. 不同植物散发不同的气味吸引 不同类型的传粉者，一些表型操作实验表明，花气味 的改变可以导致植物吸引不同的传粉者 ${ }^{[60]}$, 并因此促 进生殖隔离 ${ }^{[61]}$. 一些植物散发腐臭的味道吸引偏好这 类气味的蝇类和甲虫传粉者 ${ }^{[62]}$, 而一些植物散发气味 吸引气味感受器十分发达的鳞翅目昆虫 ${ }^{[58]}$. 一些植物 散发类似于传粉昆虫雌性个体的气味来欺骗、引诱传 粉者并与之交配 ${ }^{[63]}$. 植物与昆虫分泌的易挥发有机混 合物(VOCs)有高达 $87 \%$ 的重叠，其中芳香族化合物的 演化主要与吸引传粉者有关 ${ }^{[59]}$.

(iv) 花报酬. 植物演化出多种多样的报酬形式以 稳定植物-传粉者之间的联系, 如花蜜 ${ }^{[64]}$ 、花粉 ${ }^{[65]}$ 和树 脂 ${ }^{[6]}$. 花蜜的主要成分包括单糖(葡萄糖、果糖)和二 糖(蔗糖), 其中蔗糖所占比例常被认为是不同植物对 不同传粉者的适应 ${ }^{[67]}$. 例如, 蜂鸟传粉的花, 其花蜜中 蔗糖含量通常较高, 有利于补充蜂鸟访花的能量消耗, 而蝙蝠传粉的花, 其花蜜中常常含有丰富的果糖, 因为 传粉蝙蝠也常食用果实 ${ }^{[68]}$. 蝶类传粉的植物, 通常其 花蜜中氨基酸含量较高, 这与蝶类对高氨基酸含量花 蜜的偏好相对应 ${ }^{[69]}$. 有观点认为, 在植物与传粉昆虫 的相互作用中, 花蜜除了可以吸引传粉者、被动地适 应传粉者偏好之外, 还能主动操纵传粉昆虫行为 ${ }^{[70]}$. 例如，乌头(Aconitum spp.)花蜜中的生物碱虽然以其 苦涩的口感降低传粉者的访问频率，但其苦涩味道使 盗蜜者更加难以忍受，使得访问者更易成为传粉者而 不是盗蜜者 ${ }^{[71]}$. 花蜜中的咖啡因可以提升蜜蜂对食物

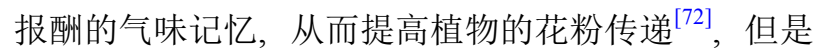
在过高浓度下, 反而降低蜜蜂的访问频率 ${ }^{[73]}$. 花粉也 是重要的报酬, 花粉中富含蛋白质和脂质, 其蛋白质 与脂质的特定比例受到蜂类传粉者的偏好 ${ }^{[74]}$.

在传粉者方面，传粉者为了获取花蜜报酬演化出 了各式各样的口器, 如鳞翅目的昆虫为从更长的花管 或花距中获取花蜜, 演化出细长的吻 ${ }^{[47]}$. 蜂类为高效 收集花粉而演化出花粉筐、花粉刷等结构 ${ }^{[75]}$. 自然界 大约有 2 万种植物具有孔裂花药或类似孔裂花药的结 构, 使花粉只能由那些可以进行震动授粉的昆虫所获 得 ${ }^{[76]}$, 具有震动授粉能力的昆虫在移出花粉时十分高 效, 而相应地, 植物演化出一系列特征, 如花药逐次开 
裂和孔裂花药等, 以减少单次花粉输出, 避免花粉储量 在短时间内耗尽 ${ }^{[77]}$. 一些昆虫为了能够获取报酬, 演 化出了极为不对称的肢体. 例如, 一种蜂类 (Rediviva longimanus)演化出极长的前肢以获取兰花(Diascia whiteheadii) 花距中的油滴 ${ }^{[78]}$.

(2) 互利共生. 互利共生是更为紧密、长期的, 在 两种不同物种之间的互惠关系.

墨西哥东部的蚂蚁 (Pseudomvrmex sp.) 依赖于豆 科金合欢属(Acacia) 植物, 以其膨大的中空棘刺作为居 所, 并以植物分泌的花外花蜜作为食物; 金合欢依赖蚂 蚁对抗的植食者、移除真菌孢子从而阻止真菌病原体 入侵植物组织 ${ }^{[4]}$. 与金合欢类似, 人们在非洲纳米比亚 发现, 在骆驼刺(Vachellia erioloba) 的棘刺中定居的蚂 蚁, 具有防御植食者的生态功能 ${ }^{[79]}$.

小丑鱼(或海葵鱼, Amphiprioninae亚科)与海葵是 经典的互惠关系. 海葵为小丑鱼提供住所和保护: 小丑 鱼身体表面有黏液覆盖的保护性外层, 可免疫海葵刺 细胞的伤害, 使其可以在海葵中定居; 小丑鱼追逐驱 赶海葵的天敌, 反过来保护海葵 ${ }^{[80,81]}$. 海洋中一共有 超过 1000 种海葵, 其中仅有 10 种与 26 种热带小丑鱼专 性共生, 与那些未与海葵建立互惠联系的近缘物种相 比, 具有互惠关系的小丑鱼有更高的物种多样化速率 以及形态演化速率 ${ }^{[82]}$.

植物与真菌的共生十分普遍, 据统计, 世界上大约 $80 \%$ 的维管植物科中都有植物与菌根真菌(arbuscular mycorrhizal, AM) 共生 ${ }^{[83]}$, 共生真菌帮助植物吸收利用 土壤中的磷、硫、氮和微量元素, 而植物为其提供碳 水化合物和其他营养物质. 植物与细菌也有共生, 较 为常见的是植物与根瘤菌的共生, 根瘤菌为植物提供 氮, 而植物为根瘤菌提供光合作用产物. 豆科植物与 共生的根瘤菌选择作用于彼此, 甚至可以呈现出基因 型对基因型的影响 ${ }^{[84]}$, 而这样的互作关系在不同环境 下还会发生改变, 呈现镶嵌式互作选择 ${ }^{[85]}$. 例如, 当土 壤氮含量充足时, 豆科植物就抑制根瘤的形成, 较少依 赖根瘤菌提供的氮, 相应根瘤菌的固氮能力也会变得 较低 ${ }^{[86,87]}$.

(3) 传粉-寄生. 一些互作关系并不是简单的对抗 或互惠, 而是互作双方在互利的同时兼具损害, 达到一 定程度的平衡. 与单纯的植物-传粉者互惠关系不同, 传粉-寄生关系还伴随对植物适合度的损害.

(i) 丝兰-丝兰蛾. 丝兰属植物 $(Y u c c a)$ 为丝兰蛾
(丝兰蛾科Prodoxidae) 提供隐蔽的产卵地以避免被捕 食, 同时丝兰蛾为丝兰提供专性特化的传粉服务 ${ }^{[88]}$. 丝兰蛾收集花粉, 并将花粉负于口器的特化附属物上, 在此过程中使柱头接受花粉. 丝兰蛾访问丝兰的花, 并 在花的子房中产卵, 孵化出的后代以发育中的种子 为食.

(ii) 榕树-榕小蜂. 榕树(Ficus spp.)及其传粉榕小 蜂构成的互作系统早在 7500 万年前就已建立, 是动植 物间最紧密的协同演化关系之一 ${ }^{[89]}$. 榕小蜂为榕树雌 花授粉, 而榕树为榕小蜂提供产卵地和哺育后代的场 所, 甚至可为幼体提供食物. 榕小蜂分为主动传粉小 蜂和被动传粉小蜂, 主动传粉小蜂胸腹板有明显的花 粉筐(pollen pocket), 前肢具花粉刷(pollen comb), 此 结构与小蜂主动收集和放置花粉的行为直接相关 ${ }^{[90] \text {; }}$ 被动传粉小蜂一般没有这些特殊结构, 也没有主动收 集、置放花粉的行为 ${ }^{[00,91]}$. 一些传粉小蜂个体采用投 机性策略, 不给榕树传粉, 而榕树则通过奖励传粉小 蜂增加其适合度, 提高自身传粉成功率, 并通过脱落 榕果或抑制小蜂后代发育, 来惩罚非传粉小蜂和投机 性传粉小蜂, 使双方关系达到平衡 ${ }^{[92,93]}$.

(iii) 叶下珠-蛾. 头细蛾(Epicephala spp.)为叶下珠 (Glochidion spp.)提供传粉服务, 但其幼虫会取食一部 分叶下珠的种子. 叶下珠属植物与头细蛾属的互作关 系在古生代就已广泛存在, 系统发生分析表明, 6 个叶 下珠属植物的演化分支都与各自独特的头细蛾属昆虫 有关联 ${ }^{[94]}$.

（4）食果-种子散布. 一些动物以植物果实为食物, 看似损害了植物的利益, 但这些动物同时也会为植物 散播种子, 对植物有利.

被子植物和食果者之间的协同演化发展了约 8000 万年 ${ }^{[95]}$. 宏观来看, 很多植物演化出了多肉的果实以 供鸟类食用并散播种子, 而很多鸟类已经适应于以果 实作为食物. 多肉果实在很多被子植物科中发生, 食 果者的类群也在众多的动物科中出现, 在 281 个已知 的陆地有花植物科中, 有一半都包含了产生多肉果实 的物种, 而 135 个已知的陆地鸟类科的 $36 \%$, 以及 107 个 已知的陆地非海生哺乳动物科的 $20 \%$, 都包含了部分 或完全的食果物种 ${ }^{[96]}$.

植物与种子散布者之间存在特征的关联. 例如, 鸟 类的颜色视觉强于哺乳动物 ${ }^{[97]}$, 而由鸟类散布种子的 植物, 其果实通常绚丽多彩, 相反由哺乳动物散布种 
子的植物, 其果实通常颜色暗淡 ${ }^{[96,98,99]}$. 果实大小与食 果鸟类嗄裂口宽度(gape width)之间有一定联系，由鸟 类散播种子的植物，常常演化出巨大的、由薄薄的富 含营养的果肉所包裹的种子，以强迫鸟类在取食时将 整个果子都吞下. 相应地, 特化的食果鸟类演化出了 更宽裂口的鸟嘴以吞下整个果子 ${ }^{[97,100 ~ 102]}$. 由迁徙食 果鸟类散布种子的植物，其最佳果实成熟时间与食果 鸟类的迁徙时间保持一致，这样不仅可以最大化种子 散布, 还可以避开种子的啃食者 ${ }^{[103 ~ 105]}$.

\section{3 竞争}

不同物种或类群一旦处于相同、相似的营养级, 生态位有重叠, 就会产生竞争关系. 在竞争关系中, 每 个物种的出现都会抑制其他物种的居群增长. 竞争伴 随竞争双方适合度的降低，其中一方终将被逐出生态 位, 因而互作物种似乎难以实现均衡与共存. 不过, 目 前有观点认为, 在竞争关系中, 协同演化的结果之一是 发生性状替代 ${ }^{[8]}$.

原产欧亚大陆的苾芥(Alliaria petiolata), 在约 150 年前引入北美, 这种入侵植物生产有毒的化感物质(allelochemical), 虽然该物质(主要是芥子式(sinigrin))在 原产地欧亚大陆对当地植物的影响并不大，但可干扰 美洲本土竞争物种的生长 ${ }^{[106,107]}$. 相应地, 本土物种演 化出了耐受这种有毒物质干扰的能力, 且葱芥入侵更 严重的区域, 本土物种的抗干扰能力越强 ${ }^{[108]}$.

在英属哥伦比亚地区的湖中，先期拓殖的海生棘 鱼(Gasterosteus aculeatus)居群演化为形态上和营养级 上的泛化者 ${ }^{[109]}$, 受后续另一批海生棘鱼入侵的影响, 本土棘鱼居群演化为淡水特化者, 而入侵居群演化为 深海特化者 ${ }^{[110]}$.

原产古巴和巴哈马群岛的棕色变色龙(Anolis sagrei)作为宠物被引入美国, 并很快转变成入侵种, 在 入侵过程中与本土的绿色变色龙(Anolis carolinensis) 竞争领土和食物. 研究人员人工引入棕色变色龙到小 岛上，发现经过短短 20 代后，与附近岛屿上没被入侵 的地区相比，本土绿色变色龙在树上栖息处的所在位 置变得更高了, 且足垫(toepad)也变得更大, 以抓握高 处不易粘附的树枝 ${ }^{[111]}$.

\section{4 寄生}

在寄生关系中, 寄生者依赖于宿主生存. 寄生者常
损害宿主的利益，而那些能够避免被寄生或能降低寄 生者危害的宿主更受选择青睐. 从这个角度来看, 寄 生者和宿主之间存在一定的对抗关系. 不过, 也有观 点认为寄生者应该演化得逐渐减少与宿主的对抗，即 “成功的”寄生者并不非常有必要演化为对宿主非常有 害 ${ }^{[112]}$.

寄生者和宿主之间的协同演化在早年就有系统发 生的证据. 例如, 研究人员使用蛋白质电泳数据对啮齿 动物和它们的皮外寄生虫(ectoparasite)建立系统树, 发 现两者之间的系统树形状和枝长非常一致, 从而认为 寄主和宿主之间的物种形成大体是同时期、有关联 的 $^{[113]}$.

寄生者与宿主之间的协同演化可以达到很快的速 度. 欧洲的兔子在19世纪初被引入澳大利亚, 而澳大利 亚缺乏兔子天敌，所以这些兔子疯狂繁殖、破坏本土 植被. 21世纪中期, 黏液病毒(myxoma virus)被引入澳 大利亚以控制兔子的居群, 这种病毒高度致命, 几乎所 有感染病毒的兔子在短期内即会死亡, 随后, 病毒和兔 子之间发生了协同演化，病毒变得对于兔子而言不那 么致命了, 因为当兔子数量降低时, 那些允许兔子宿 主活得更长的病毒的突变系就更受偏好, 从而增加了 在宿主死亡之前传播到其他兔子的机会. 虽然之后许 多兔子仍然死于感染，大多数受感染的个体存活时间 却大幅提高 ${ }^{[114]}$.

一些鸟类将卵产在其他鸟类的巢穴中，幼体由被 寄生的鸟类抚育, 是为巢穴寄生 (brood parasite) ${ }^{[115]}$. 寄 生鸟类和宿主鸟类之间的对抗，不仅发生在卵期( egg stage), 也发生在雏鸟期(chick/nestling stage). 为了避 免抚养与自己没有亲缘关系的寄生鸟类幼体，宿主鸟 类演化出分辨非本巢鸟蛋, 并弃于巢外的能力, 相应 地，巢穴寄生鸟类演化出一系列对策，包括拟态宿主 鸟蛋、伪装鸟蛋、增厚蛋壳等 ${ }^{[116 ~ 118]}$. 寄生鸟类与宿 主鸟类存在着军备竞赛. 例如, 不同宿主鸟类对寄生 鸟蛋的丢弃程度不同, 而在丢弃程度更高的鸟巢中, 寄生鸟类大杜鹃(Cuculus canorus)的鸟蛋拟态程度也 越高 ${ }^{[119]}$. 此外，尽管寄生鸟类的蛋不断拟态宿主鸟类 的蛋, 宿主鸟类蛋的外观也可不断转变、“逃离”原有 寄生鸟蛋的外观, 或使鸟蛋外观多种多样, 增加寄生 鸟类拟态的难度. 例如, 大杜鹃的宿主鸟类物种, 其识 别、丢弃寄生鸟蛋的能力越强，则相应的同种鸟类不 同鸟巢中宿主鸟蛋外观多样性也越高 ${ }^{[120]}$. 


\section{5 拟态}

一些蝴蝶在幼虫期可以将植物食物中的有毒化学 物质存储起来, 使自身变得很难吃, 因而具有该类蝴蝶 颜色样式的蝴蝶, 被捕食的几率大幅降低, 该类颜色样 式即为“原型”(model), 其他种类的蝴蝶以此演化出类 似的颜色模式，以模拟那些难吃的蝴蝶，成为“拟 型” (mimic) ${ }^{[121,122]}$. 拟型的存在, 对原型是不利的, 因为 一旦拟型变得十分常见, 则原型所对应的难吃的记忆 就不再专性，所以捕食者也会开始攻击原型。尽管原 型仍然很难吃，但即使被捕食者“品尝”一下，也会使 蝴蝶付出生命的代价. 因此, 拟态也可推动协同演化, 即拟型向着原型的颜色样式发生演化，而原型则向着 远离拟型的颜色样式发生演化.

生物对生物的拟态分为缪氏拟态和贝氏拟态. 在 缪氏拟态(Müllerian mimicry)中, 原型物种和拟型物种 对捕食者而言都是难吃的或有毒的，因此双方颜色样 式的演化都有机会受到影响. 例如，在常被用作协同 演化范例的Heliconius 属蝴蝶中, H. erato和H. melpomene 是研究得最为详细的两个物种, 通常情况下, $H$. melpomene模拟 H. erato 的翅膀图样. 两物种之间是否 发生协同演化, 一直受到质疑. 基于前人对翅膀颜色样 式控制基因的测序，近期一项研究探讨了这两种蝴蝶 的系统发生分枝模式、物种分化次数以及谱系地理学 历史，验证了两者翅膀样式之间的确存在严格的协同 演化关系 ${ }^{[123]}$.

在贝氏拟态(Batesian mimicry)中, 拟型通过模拟 有毒原型的警戒色来对抗捕食者. 例如，珊瑚蛇( $\mathrm{Fa}$ mily：Elapidae)是一类广泛分布于新世界和旧世界毒 蛇的统称，但有许多无毒蛇的花纹与珊瑚蛇类似. 研 究人员通过整合来自新世界地区的蛇类物种的分布、

形态、系统发生数据, 以系统发生分析为框架, 重建无 毒蛇拟态的起源和体色样式的演化速率，发现无毒蛇 体色样式的转变, 与珊瑚蛇在整个西半球的空间上和 纵贯4000万年的时间尺度上高度关联 ${ }^{[124]}$.

\section{5 协同演化的地理镶嵌}

无论是物种间还是类群间的协同演化，发生互作 的基本单元仍然是居群. 居群在空间的分布上具有异 质性，形成地理上的镶嵌，因而物种间的相互作用实
质上是众多异质居群互作的集合，物种间的协同演化 方向也是各个居群交互选择合力的体现 ${ }^{[125 ~ 129]}$. 协同 演化的地理镶嵌理论(geographic mosaic theory of coevolution, GMTC)的提出正是基于这样的 3 个前提: 物 种是居群的遗传组合、互作物种通常存在镶嵌式的地 理分布、物种之间的互作关系根据环境不同而有所不 同 $^{[125,130,131]}$.

传统的协同进化理论假设物种之间的关系在空间 分布上是静态的, 而协同演化的地理镶嵌理论认为, 协 同演化具有空间变异，且以 3 个过程作为主要驱动力: ( i ) 协同演化冷点和热点的产生. 种间互作在地理各 异的情况下多种多样, 互作两物种彼此施加了选择压 力的区域即为热点, 反之为冷点. (ii) 镶嵌式选择作 用. 互作物种受到的自然选择, 在不同的环境间各不相 同. (iii) 特征再混合. 通过突变、基因流、随机遗传漂 变、灭绝并伴随本土居群的拓殖等过程导致本土物种 的失配(mismatch)或适应性降低(maladaptation). 这 3 个 过程的结合, 持续不断地改变了本土居群之内和居群 之间的基因型分布 ${ }^{[132,133]}$.

北美落基山区的红松鼠(Tamiascirus hudsonicus)、红交潒鸟(Lixia curvirostra complex)与美国黑松 (Pinus contorta ssp. latifolia)互作系统是协同演化地理 镶嵌理论的经典案例. 在大多区域, 松树种子被松鼠和 交喙鸟取食, 松树演化出更重的球果、更少的种子、 更薄的鳞片以对抗松鼠的取食, 而未见演化出抵御鸟 类的特征，同时这些区域的交喙鸟也未见演化出利于 取食松果的特征，此类区域即为松树与交潒鸟的协同 演化冷点; 在一些小的较为隔离的区域中, 交喙鸟是 松树唯一的取食者, 松树球果则演化出更厚甚至重叠 的鳞片以抵御交喙鸟的取食，同时此区域的交喙鸟演 化出更厚、更短、更直的喙来破开鳞片，这些区域即 为松树与交嚎鸟的协同演化热点地区，从而，同一互 作系统在空间变异中呈现出了地理镶嵌式的选择强 度 ${ }^{[134,135]}$.

\section{6 结语}

协同演化概念的起源与创立，已有一百多年的历 史，其应用范围也经历了从狭义到广义，从广义到超 出生物学意义的历程. 虽然人们常常扩大或误用“coevolution”概念的范畴，若以 3 条基本特征为准，即协同 
互作、交互选择和协同系统发生，则仍可认为它们是 协同演化的关系.

演化, 这一术语本身伴随着强烈的时间属性. 演化 本身可能时间漫长，自然的选择经历了数万年的时间; 但一些人工选择也发现了快速的协同演化，如杂草抗 除草剂、动物和人在个体生命周期内产生的耐药性. 当时间尺度超出人类观测的极限，那么将时间变化转 换为空间变化是探究自然选择的一个合理有效的选 择. 20 世纪 90 年代以来兴起的协同演化的地理镶嵌理 论是这一思路的具体体现. 自然选择最终作用于居群 的等位基因频率，协同演化的地理镶嵌理论反映的是 最贴近真实的物种互作图景：处在不同演化阶段的物 种居群在不同区域发生互作，不同区域对彼此的选择 强度有所差异，宏观整合的各个镶嵌式的地理区域汇
总构成了真正的物种间的协同互作，反应了物种间随 时间流逝而发生的协同演化.

生态学中的许多基本关系，都可能包含了协同演 化的历史. 在众多生态关系中，互惠关系是最易形成 协同演化的. 这是因为, 互惠之外的关系, 如捕食、竞 争、寄生、拟态等，都或多或少包含了一方对另一方 适合度的损害, 且无论环境的动荡, 在系统内部, 互作 双方此消彼长之下，其中一方在漫长的演化历史中消 亡. 而互惠关系, 则较少会受到系统内力的影响. 当然, 将物种与物种之间的关系简单归为单一的关系颇具难 度. 许多物种之间的互作, 既是互利的, 又是互相(或单 方面)损害的. 正是这些复杂的生态关系, 最终导致了 可能的协同多样化，形成当前地球上生物多样化的 格局.

\section{参考文献}

1 Darwin C. On the Origin of Species by Means of Natural Selection. London: John Murray, 1859

2 Ehrlich P R, Raven P H. Butterflies and plants: a study in coevolution. Evolution, 1964, 18: 586-608

3 Janzen D H. When is it coevolution? Evolution, 1980, 34: 611-612

4 Janzen D H. Coevolution of mutualism between ants and acacias in Central America. Evolution, 1966, 20: 249-275

5 Fox L R. Diffuse coevolution within complex communities. Ecology, 1988, 69: 906-907

6 Althoff D M, Segraves K A, Johnson M T J. Testing for coevolutionary diversification: linking pattern with process. Trends Ecol Evol, 2014, 29: $82-89$

7 Pollock D D, Thiltgen G, Goldstein R A. Amino acid coevolution induces an evolutionary Stokes shift. Proc Natl Acad Sci USA, 2012, 109: E1352-E1359

8 Hembry D H, Yoder J B, Goodman K R. Coevolution and the diversification of life. Am Nat, 2014, 184: 425-438

9 Lewis S L, Maslin M A. Defining the anthropocene. Nature, 2015, 519: 171-180

10 Waters C N, Zalasiewicz J, Summerhayes C, et al. The Anthropocene is functionally and stratigraphically distinct from the Holocene. Science, 2016, 351: aad2622

11 Dobretsov N L, Kolchanov N A, Suslov V V. On the early stages of the evolution of the geosphere and biosphere. Paleontol J, 2006, 40: S407S424

12 Algeo T J, Marenco P J, Saltzman M R. Co-evolution of oceans, climate, and the biosphere during the 'Ordovician Revolution': a review. Palaeogeogr Palaeoclimatol Palaeoecol, 2016, 458: 1-11

13 Kormendy J, Ho L C. Coevolution (or not) of supermassive black holes and host galaxies. Annu Rev Astron Astrophys, 2013, 51: 511-653

14 Heckman T M, Best P N. The coevolution of galaxies and supermassive black holes: insights from surveys of the contemporary universe. Annu Rev Astron Astrophys, 2014, 52: 589-660

15 Suchan T, Alvarez N. Fifty years after Ehrlich and Raven, is there support for plant-insect coevolution as a major driver of species diversification? Entomol Exp Appl, 2015, 157: 98-112

16 Abrams P A. The evolution of predator-prey interactions: theory and evidence. Annu Rev Ecol Syst, 2000, 31: 79-105

17 Brown C M, Henderson D M, Vinther J, et al. An exceptionally preserved three-dimensional armored dinosaur reveals insights into coloration and cretaceous predator-prey dynamics. Curr Biol, 2017, 27: 1-8

18 West K, Cohen A, Baron M. Morphology and behavior of crabs and gastropods from Lake Tanganyika, Africa: implications for lacustrine predator-prey coevolution. Evolution, 1991, 45: 589-607 
19 Weigand A M. The Volkswagen Foundation Lake Malawi Field School 2012 Consortium, Plath M. Prey preferences in captivity of the freshwater crab Potamonautes lirrangensis from Lake Malawi with special emphasis on molluscivory. Hydrobiologia, 2014, 739: 145-153

20 Hague M T J, Feldman C R, Brodie Jr. E D, et al. Convergent adaptation to dangerous prey proceeds through the same first-step mutation in the garter snake Thamnophis sirtalis. Evolution, 2017, 71: 1504-1518

21 Holding M L, Biardi J E, Gibbs H L. Coevolution of venom function and venom resistance in a rattlesnake predator and its squirrel prey. Proc R Soc B, 2016, 283: 20152841

22 Futuyma D J, Agrawal A A. Macroevolution and the biological diversity of plants and herbivores. Proc Natl Acad Sci USA, 2009, 106: 1805418061

23 Johnson S D. Carrion flowers. Curr Biol, 2016, 26: R556-R558

24 Wing S L, Herrera F, Jaramillo C A, et al. Late Paleocene fossils from the Cerrejon Formation, Colombia, are the earliest record of Neotropical rainforest. Proc Natl Acad Sci USA, 2009, 106: 18627-18632

25 Filho E B S, Adami-Rodrigues K, Lima F J, et al. Evidence of plant-insect interaction in the early Cretaceous Flora from the Crato Formation, Araripe Basin, Northeast Brazil. Historical Biol, 2017, 54: 1-12

26 Zhu Y R, Yang M, Vamosi J C, et al. Feeding the enemy: loss of nectar and nectaries to herbivores reduces tepal damage and increases pollinator attraction in Iris bulleyana. Biol Lett, 2017, 13: 20170271

27 Agrawal A A. Overcompensation of plants in response to herbivory and the by-product benefits of mutualism. Trends Plant Sci, 2000, 5: 309313

28 Chen Y Z, Huang S Q. Red young leaves have less mechanical defence than green young leaves. Oikos, 2013, 122: 1035-1041

29 Niu Y, Chen G, Peng D L, et al. Grey leaves in an alpine plant: a cryptic colouration to avoid attack? New Phytol, 2014, 203: 953-963

30 War A R, Paulraj M G, Ahmad T, et al. Mechanisms of plant defense against insect herbivores. Plant Signal Behav, 2012, 7: 1306-1320

31 Mithöfer A, Boland W. Plant defense against herbivores: chemical aspects. Annu Rev Plant Biol, 2012, 63: 431-450

32 Dicke M, Van Beek T A, Posthumus M A, et al. Isolation and identification of volatile kairomone that affects acarine predatorprey interactions Involvement of host plant in its production. J Chem Ecol, 1990, 16: 381-396

33 Huang T, Jander G, de Vos M. Non-protein amino acids in plant defense against insect herbivores: representative cases and opportunities for further functional analysis. Phytochemistry, 2011, 72: 1531-1537

34 Schuman M C, Baldwin I T. The layers of plant responses to insect herbivores. Annu Rev Entomol, 2016, 61: 373-394

35 Dyer L A, Philbin C S, Ochsenrider K M, et al. Modern approaches to study plant-insect interactions in chemical ecology. Nat Rev Chem, 2018, 2: $50-64$

36 Opitz S E W, Müller C. Plant chemistry and insect sequestration. Chemoecology, 2009, 19: 117-154

37 Winde I, Wittstock U. Insect herbivore counteradaptations to the plant glucosinolate-myrosinase system. Phytochemistry, 2011, 72: 1566-1575

38 Wittstock U, Halkier B A. Glucosinolate research in the Arabidopsis era. Trends Plant Sci, 2002, 7: 263-270

39 Scott J G, Wen Z. Cytochromes P450 of insects: the tip of the iceberg. Pest Manag Sci, 2001, 57: 958-967

40 Cardinal S, Danforth B N. Bees diversified in the age of eudicots. Proc R Soc B, 2013, 280: 20122686

41 Lidgard S, Crane P R. Quantitative analyses of the early angiosperm radiation. Nature, 1988, 331: 344-346

42 Lunau K. Adaptive radiation and coevolution? pollination biology case studies. Org Diversity Evol, 2004, 4: 207-224

43 Luo S X, Zhang L J, Yuan S, et al. The largest early-diverging angiosperm family is mostly pollinated by ovipositing insects and so are most surviving lineages of early angiosperms. Proc R Soc B, 2018, 285: 20172365

44 Paudel B R, Shrestha M, Burd M, et al. Coevolutionary elaboration of pollination-related traits in an alpine ginger (Roscoea purpurea) and a tabanid fly in the Nepalese Himalayas. New Phytol, 2016, 211: 1402-1411

45 Anderson B, Johnson S D. The geographical mosaic of coevolution in a plant-pollinator mutualism. Evolution, 2008, 62: 220-225

46 Anderson B, Johnson S D. Geographical covariation and local convergence of flower depth in a guild of fly-pollinated plants. New Phytol, 2009, 182: $533-540$

47 Whittall J B, Hodges S A. Pollinator shifts drive increasingly long nectar spurs in columbine flowers. Nature, 2007, 447: 706-709

48 Netz C, Renner S S. Long-spurred Angraecum orchids and long-tongued sphingid moths on Madagascar: a time frame for Darwin's predicted Xanthopan/Angraecum coevolution. Biol J Linnean Soc, 2017, 122: 469-478

49 Shrestha M, Dyer A G, Boyd-Gerny S, et al. Shades of red: bird-pollinated flowers target the specific colour discrimination abilities of avian 
vision. New Phytol, 2013, 198: 301-310

50 Lind O, Henze M J, Kelber A, et al. Coevolution of coloration and colour vision? Phil Trans R Soc B, 2017, 372: 20160338

51 Schiestl F P, Johnson S D. Pollinator-mediated evolution of floral signals. Trends Ecol Evol, 2013, 28: 307-315

52 Renoult J P, Valido A, Jordano P, et al. Adaptation of flower and fruit colours to multiple, distinct mutualists. New Phytol, 2014, 201: 678-686

53 Smith S D, Goldberg E E. Tempo and mode of flower color evolution. Am J Bot, 2015, 102: 1014-1025

54 Peitsch D, Fietz A, Hertel H, et al. The spectral input systems of hymenopteran insects and their receptor-based colour vision. J Comp Physiol A, 1992, 170: 23-40

55 Odeen A, Hastad O. Complex distribution of avian color vision systems revealed by sequencing the SWS1 opsin from total DNA. Mol Biol Evol, 2003, 20: 855-861

56 Reisenman C E, Giurfa M. Chromatic and achromatic stimulus discrimination of long wavelength (red) visual stimuli by the honeybee Apis mellifera. Arthropod-Plant Interact, 2008, 2: 137-146

57 Moyroud E, Wenzel T, Middleton R, et al. Disorder in convergent floral nanostructures enhances signalling to bees. Nature, 2017, 550: 469-474

58 Raguso R A. Wake up and smell the roses: the ecology and evolution of floral scent. Annu Rev Ecol Evol Syst, 2008, 39: 549-569

59 Schiestl F P. The evolution of floral scent and insect chemical communication. Ecol Lett, 2010, 13: 643-656

60 Shuttleworth A, Johnson S D. The missing stink: sulphur compounds can mediate a shift between fly and wasp pollination systems. Proc R Soc B, 2010, 277: 2811-2819

61 Waelti M O, Muhlemann J K, Widmer A, et al. Floral odour and reproductive isolation in two species of Silene. J Evol Biol, 2008, 21: 111-121

62 Johnson M T J, Campbell S A, Barrett S C H. Evolutionary interactions between plant reproduction and defense against herbivores. Annu Rev Ecol Evol Syst, 2015, 46: 191-213

63 Schiestl F P, Peakall R, Mant J G, et al. The chemistry of sexual deception in an orchid-wasp pollination system. Science, 2003, 302: 437-438

64 De la Barrera E, Nobel P S. Nectar: properties, floral aspects, and speculations on origin. Trends Plant Sci, 2004, 9: 65-69

65 Russell A L, Golden R E, Leonard A S, et al. Bees learn preferences for plant species that offer only pollen as a reward. Behav Ecol, 2015, 27: $731-740$

66 Armbruster W S, Gong Y B, Huang S Q. Are pollination "syndromes" predictive? Asian Dalechampia fit neotropical models. Am Nat, 2011, 178: $135-143$

67 Abrahamczyk S, Kessler M, Hanley D, et al. Pollinator adaptation and the evolution of floral nectar sugar composition. J Evol Biol, 2017, 30: $112-127$

68 Willmer P. Pollination and Floral Ecology. New Jersey: Princeton University Press, 2011

69 Mevi-Schutz J, Erhardt A. Amino acids in nectar enhance butterfly fecundity: a long-awaited link. Am Nat, 2005, 165: 411-419

70 Pyke G H. Plant-pollinator co-evolution: it's time to reconnect with optimal foraging theory and evolutionarily stable strategies. Perspect Plant Ecol Evol Syst, 2016, 19: 70-76

71 Barlow S E, Wright G A, Ma C, et al. Distasteful nectar deters floral robbery. Curr Biol, 2017, 27: 2552-2558.e3

72 Thomson J D, Draguleasa M A, Tan M G. Flowers with caffeinated nectar receive more pollination. Arthropod-Plant Interact, 2015, 9: 1-7

73 Stevenson P C, Nicolson S W, Wright G A. Plant secondary metabolites in nectar: impacts on pollinators and ecological functions. Funct Ecol, 2017, 31: 65-75

74 Vaudo A D, Patch H M, Mortensen D A, et al. Macronutrient ratios in pollen shape bumble bee (Bombus impatiens ) foraging strategies and floral preferences. Proc Natl Acad Sci USA, 2016, 113: E4035-E4042

75 Goulson D. Bumblebees: Their Behaviour and Ecology. Oxford: Oxford University Press, 2003

76 Buchmann S L. Buzz pollination in angiosperms. In: Jones C E, Little J R, Eds. Handbook of Experimental Pollination Biology. New York: Van Nostrand Reinhold, 1983. 73-113

77 De Luca P A, Vallejo-Marín M. What's the 'buzz’ about? The ecology and evolutionary significance of buzz-pollination. Curr Opin Plant Biol, 2013, 16: 429-435

78 Pauw A, Kahnt B, Kuhlmann M, et al. Long-legged bees make adaptive leaps: linking adaptation to coevolution in a plant-pollinator network. Proc R Soc B, 2017, 284: 20171707

79 Campbell H, Townsend I R, Fellowes M D E, et al. Thorn-dwelling ants provide antiherbivore defence for camelthorn trees, Vachellia erioloba, in Namibia. Afr J Ecol, 2013, 51: 590-598 
80 Ollerton J, McCollin D, Fautin D G, et al. Finding NEMO: nestedness engendered by mutualistic organization in anemonefish and their hosts. Proc R Soc B, 2007, 274: 591-598

81 Ricciardi F, Boyer M, Ollerton J. Assemblage and interaction structure of the anemonefish-anemone mutualism across the Manado region of Sulawesi, Indonesia. Environ Biol Fish, 2010, 87: 333-347

82 Litsios G, Sims C A, Wüest R O, et al. Mutualism with sea anemones triggered the adaptive radiation of clownfishes. BMC Evol Biol, 2012, 12: 212

83 Schüßler A, Schwarzott D, Walker C. A new fungal phylum, the Glomeromycota: phylogeny and evolution. Mycol Res, 2001, 105: 1413-1421

84 Burghardt L T, Guhlin J, Chun C L, et al. Transcriptomic basis of genome by genome variation in a legume-rhizobia mutualism. Mol Ecol, 2017, 26: $6122-6135$

85 Cauwenberghe J V, Visch W, Michiels J, et al. Selection mosaics differentiate Rhizobium-host plant interactions across different nitrogen environments. Oikos, 2016, 125: 1755-1761

86 Naudin C, Corre-Hellou G, Voisin A S, et al. Inhibition and recovery of symbiotic N2 fixation by peas (Pisum sativum L.) in response to shortterm nitrate exposure. Plant Soil, 2011, 346: 275-287

87 Weese D J, Heath K D, Dentinger B T M, et al. Long-term nitrogen addition causes the evolution of less-cooperative mutualists. Evolution, 2015, 69: 631-642

88 Althoff D M. Specialization in the yucca-yucca moth obligate pollination mutualism: a role for antagonism? Am J Bot, 2016, 103: 1803-1809

89 Cruaud A, Rønsted N, Chantarasuwan B, et al. An extreme case of plant-insect codiversification: figs and fig-pollinating wasps. Systatic Biol, 2012, 61: 1029-1047

90 Kjellberg F, Jousselin E, Bronstein J L, et al. Pollination mode in fig wasps: the predictive power of correlated traits. Proc R Soc B, 2001, 268: $1113-1121$

91 Chen Y, Li H Q, Liu M, et al. Species-specificity and coevolution of figs and their pollinating wasps (in Chinese). Biodiv Sci, 2010, 18: 1-10 [陈 艳, 李宏庆, 刘敏, 等. 榕-传粉榕小蜂间的专一性与协同进化. 生物多样性, 2010, 18: 1-10]

$92 \mathrm{Lu} \mathrm{C}$, Geng Y P, Wang R W. Asymmetric interaction and its effects on the meta-population dynamics in co-evolved fig-fig wasps systems (in Chinese). Biodiv Sci, 2012, 20: 264-269 [路程, 耿宇鹏, 王瑞武. 榕树-榕小蜂协同进化中的非对称性相互作用及其集合种群效应. 生物多 样性, 2012, 20: 264-269]

93 Guan J M, Peng Y Q, Yang D R. Host sanctions in fig-fig wasp mutualism (in Chinese). Biodiv Sci, 2007, 15: 626-632 [管俊明, 彭艳琼, 杨大 荣. 榕-蜂互惠关系中榕树对未传粉榕小蜂的惩罚效应. 生物多样性, 2007, 15: 626-632]

94 Luo S X, Yao G, Wang Z, et al. A novel, enigmatic basal leafflower moth lineage pollinating a derived leafflower host illustrates the dynamics of host shifts, partner replacement, and apparent coadaptation in intimate mutualisms. Am Nat, 2017, 189: 422-435

95 Eriksson O. Evolution of angiosperm seed disperser mutualisms: the timing of origins and their consequences for coevolutionary interactions between angiosperms and frugivores. Biol Rev, 2016, 91: 168-186

96 Herrera C M. Seed dispersal by vertebrates. In: Herrera C M, Pellmyr O, Eds. Plant-animal Interactions: An Evolutionary Approach. UK, Oxford: Blackwell, 2002. 185-208

97 Valido A, Schaefer H M, Jordano P. Colour, design and reward: phenotypic integration of fleshy fruit displays. J Evol Biol, 2011, 24: 751-760

98 Cazetta E, Schaefer H M, Galetti M. Why are fruits colorful? The relative importance of achromatic and chromatic contrasts for detection by birds. Evol Ecol, 2009, 23: 233-244

99 Amico G C, Rodriguez-Cabal M A, Aizen M A. Geographic variation in fruit colour is associated with contrasting seed disperser assemblages in a South-Andean mistletoe. Ecography, 2011, 34: 318-326

100 Wheelwright N T. Fruit-size, gape width, and the diets of fruit-eating birds. Ecology, 1985, 66: 808-818

101 Galetti M, Guevara R, Côrtes M C, et al. Functional extinction of birds drives rapid evolutionary changes in seed size. Science, 2013, 340: $1086-1090$

102 Burns K C. What causes size coupling in fruit-frugivore interaction webs? Ecology, 2013, 94: 295-300

103 Stiles E W. Patterns of fruit presentation and seed dispersal in bird-disseminated woody plants in the eastern deciduous forest. Am Nat, 1980, 116: $670-688$

104 Noma N, Yumoto T. Fruiting phenology of animal-dispersed plants in response to winter migration of frugivores in a warm temperate forest on Yakushima Island, Japan. Ecol Res, 1997, 12: 119-129 
105 Boulay R, Carro F, Soriguer R C, et al. Synchrony between fruit maturation and effective dispersers' foraging activity increases seed protection against seed predators. Proc R Soc B, 2007, 274: 2515-2522

106 Callaway R M, Cipollini D, Barto K, et al. Novel weapons: invasive plant suppresses fungal mutualists in America but not in its native Europe. Ecology, 2008, 89: 1043-1055

107 Lankau R A, Nuzzo V, Spyreas G, et al. Evolutionary limits ameliorate the negative impact of an invasive plant. Proc Natl Acad Sci USA, 2009, 106: $15362-15367$

108 Lankau R A. Coevolution between invasive and native plants driven by chemical competition and soil biota. Proc Natl Acad Sci USA, 2012, 109: $11240-11245$

109 Schluter D. Frequency dependent natural selection during character displacement in sticklebacks. Evolution, 2003, 57: 1142-1150

110 Schluter D, Marchinko K B, Barrett R D H, et al. Natural selection and the genetics of adaptation in threespine stickleback. Phil Trans R Soc B, 2010, 365: 2479-2486

111 Stuart Y E, Campbell T S, Hohenlohe P A, et al. Rapid evolution of a native species following invasion by a congener. Science, 2014, 346: 463466

112 May R M, Anderson R M. Epidemiology and genetics in the coevolution of parasites and hosts. Proc R Soc Lond B, 1983, 219: 281-313

113 Hafner M S, Nadler S A. Phylogenetic trees support the coevolution of parasites and their hosts. Nature, 1988, 332: 258-259

114 Elsworth P, Cooke B D, Kovaliski J, et al. Increased virulence of rabbit haemorrhagic disease virus associated with genetic resistance in wild Australian rabbits (Oryctolagus cuniculus). Virology, 2014, 464-465: 415-423

115 Rothstein S I. A model system for coevolution: avian brood parasitism. Annu Rev Ecol Syst, 1990, 21: 481-508

116 Spottiswoode C N, Stevens M. How to evade a coevolving brood parasite: egg discrimination versus egg variability as host defences. Proc R Soc B, 2011, 278: 3566-3573

117 Stoddard C M, Marshall K L A, Kilner R M. Imperfectly camouflaged avian eggs: artefact or adaptation? Avian Biol Res, 2011, 4: 196-213

118 Feeney W E, Welbergen J A, Langmore N E. The frontline of avian brood parasite-host coevolution. Animal Behaviour, 2012, 84: 3-12

119 Stoddard M C, Stevens M. Pattern mimicry of host eggs by the common cuckoo, as seen through a bird's eye. Proc R Soc B, 2010, 277: 13871393

120 Soler J J, Pape Møller A. A comparative analysis of the evolution of variation in appearance of eggs of European passerines in relation to brood parasitism. Behav Ecol, 1996, 7: 89-94

121 Estabrook G F, Jespersen D C. Strategy for a predator encountering a model-mimic system. Am Nat, 1974, 108: 443-457

122 Malcolm S B. Mimicry: status of a classical evolutionary paradigm. Trends Ecol Evol, 1990, 5: 57-62

123 Cuthill J F H, Charleston M. Wing patterning genes and coevolution of Müllerian mimicry in Heliconius butterflies: support from phylogeography, cophylogeny, and divergence times. Evolution, 2015, 69: 3082-3096

124 Rabosky A R D, Cox C L, Rabosky D L, et al. Coral snakes predict the evolution of mimicry across New World snakes. Nat Commun, 2016, 7: 11484

125 Thompson J N. The Coevolutionary Process. Chicago: Chicago University Press, 1994

126 Case T J, Taper M L. Interspecific competition, environmental gradients, gene flow, and the coevolution of species’ borders. Am Nat, 2000, 155: 583-605

127 Gomulkiewicz R, Drown D M, Dybdahl M F, et al. Dos and don'ts of testing the geographic mosaic theory of coevolution. Heredity, 2007, 98: $249-258$

128 Hochberg M E, Gomulkiewicz R, Holt R D, et al. Weak sinks could cradle mutualistic symbioses-strong sources should harbour parasitic symbioses. J Evol Biol, 2000, 13: 213-222

129 Nuismer S L, Thompson J N, Gomulkiewicz R. Coevolutionary clines across selection mosaics. Evolution, 2000, 54: 1102-1115

130 Thompson J N. The Geographic Mosaic of Coevolution. Chicago: University of Chicago Press, 2005

131 Thompson J N. Relentless Evolution. Chicago: University of Chicago Press, 2013

132 Thompson J N. Specific hypotheses on the geographic mosaic of coevolution. Am Nat, 1999, 153: S1-S14

133 Gomulkiewicz R, Thompson J N, Holt R D, et al. Hot spots, cold spots, and the geographic mosaic theory of coevolution. Am Nat, 2000, 156: $156-174$

134 Benkman C W, Holimon W C, Smith J W. The influence of a competitor on the geographic mosaic of coevolution between crossbills and 


\title{
The development, misuse and evidence of the concept "coevolution"
}

\section{TONG ZeYu \& HUANG ShuangQuan}

\author{
Institute of Ecology and Evolution, School of Life Sciences, Central China Normal University, Wuhan 430079
}

\begin{abstract}
Natural selection is one of the most important components of organic evolution, while the concept of coevolution proposes that reciprocal selection generated by interacting species could also drive their organic evolution. Formally raised in 1964, the concept of coevolution has extensively developed, but been misused or even abused. Although continuously emerging studies have verified that some ecological interactions are indeed coevolution, queries of whether it is an acceptable concept remain. To clarify the precise use of the concept, we list and re-induce mistakes in using or misunderstanding of coevolution. Here a concise principle for correct usage is suggested, i.e. confirming three aspects: species interaction, reciprocal selection as well as co-phylogenesis. Bibliometric methods are applied to quantify the development of the coevolution concept, showing the bursting expansion of the term "coevolution" into study fields beyond evolutionary biology, and revealing that current usage of "coevolution" still remains within a reasonable range. Currently, studies of ecological interactions are commonly considered as in the coevolution field. Differences and commonsense of the other six related terms, namely coadaptation, cooperation, interaction, interplay, mutualism and symbiosis, are briefly discussed. Finally, case studies of frontier topics of coevolution, geographic mosaic theory of coevolution, are introduced. A better understanding of the coevolution concept could be achieved if misuse or abuse is avoided. This paper may provide references for future coevolution studies.
\end{abstract}

ecological relationships, interaction, coevolution, geographic mosaic theory of coevolution, evolution of concept

doi: $10.1360 / \mathrm{N} 052018-00221$ 